\title{
Morphological alteration of seminiferous tubules of testes of Wistar rat offspring exposed to alcohol during pregnancy and/or lactation
}

\author{
J. E. ONU ${ }^{1 *}$, B. O. OKE ${ }^{2}$, P. C. OZEGBE ${ }^{2}$ and J. O. OYEWALE ${ }^{3}$ \\ ${ }^{I}$ Department of Veterinary Anatomy, Usmanu Danfodiyo University, Sokoto, Nigeria. \\ ${ }^{2}$ Department of Veterinary Anatomy, University of Ibadan, Nigeria. \\ ${ }^{3}$ Physiology, Pharmacology and Biochemistry, University of Ibadan, Nigeria. \\ ${ }^{*}$ Corresponding author; E-mail: josephatedoga@yahoo.co.uk
}

\begin{abstract}
This paper presents the effects of alcohol on the morphology of seminiferous tubules of testes of Wistar rat offspring exposed to alcohol during pregnancy and/or lactation. Seventy five adult female Wistar rats divided into 3 groups of 25 each and their offsprings were used. The offspring of group 1 served as control, those of group 2 were exposed to $2 \mathrm{~g} / \mathrm{kg}$ body weight of $30 \%$ ethanol $(\mathrm{v} / \mathrm{v})$ during pregnancy and lactation (APL) while those of group 3 were exposed to the same dose of ethanol during lactation only (AL). At Day (D) 7, 14, 21, 35 and 49 of postnatal life, 5 male offspring were randomly selected from the three groups and sacrificed. After the sacrifice, the two testes were dissected out and then prepared for routine histological evaluation. The result of the study showed that the seminiferous epithelia of the testes of the alcohol-exposed groups were characterized by few adluminal as well as defoliated germ cells. The result further showed significant reduction $(\mathrm{P}<0.05)$ in the diameter of the seminiferous tubules of the testes in alcohol-exposed groups. This histomorphometric effects which persisted into adulthood may have implications on the fertility of male offspring of dams who abuse alcohol during pregnancy and/or lactation.
\end{abstract}

(c) 2014 International Formulae Group. All rights reserved.

Keywords: Alcohol, pregnancy, lactation, testes, histomorphometry, Wistar rats.

\section{INTRODUCTION}

Prenatal alcohol exposure has marked effects on the development of the foetus including the foetal hypothalamic-pituitarygonadal axes in the offspring (Lam et al., 2000). It has been observed that alcohol consumption during pregnancy leads to Foetal Alcohol Syndrome (FAS) in humans and other species which is characterized by growth deficiency, microcephaly and central nervous system dysfunction (May, 2001). Prenatal alcohol exposure also leads a condition called Fetal Alcohol Spectrum Disorders (FASD). This condition is an umbrella term which describes a continuum of permanent birth defects caused by maternal consumption of alcohol during pregnancy, which includes, but is not limited to Fetal 
Alcohol Syndrome (FAS) (Astley, 2004). The brains of affected individuals may have less volume and fewer neurons that are able to function correctly, leading to long-term problems in learning and behaviour. This alcohol-induced reduction in brain volume and neuronal loss may be attributed to alcohol-induced neurodegeneration via programmed cell death (apoptosis) as observed by Li and Kim (2003) in gonocytes. This could be as a result of the effect of alcohol on neurotrophins, a family of peptides that influence the growth, development and functional plasticity of the fetal brain. Neurotrophins are produced in the nervous system and are critical for normal development of the brain (Berman, 2014). According to Beaulieu and Rasmassen (2013) who used Magnetic Resonance Imaging (MRI) to see the kind of changes that occur in the brain of patients with FASD as they age, there was decreased brain weight and white matter composition. White matter forms connections between various regions of the brain and usually develops significantly during childhood and adolescence which corresponds to postnatal period in rats (Spear, 2000). In man, the brain growth spurt which is a period during which the brain is particularly vulnerable to environmental influences begins at about mid-pregnancy and peaks around birth. The corresponding period in laboratory animals such as rats that are frequently used as study subjects typically spans postnatal days 30-50 (i.e., PD30PD50). In both humans and animal models, the brain undergoes extensive remodelling during this period. New connections among neurons are formed and at the same time, a substantial number of existing connections are lost (Spear 2000). It is probable that alcohol administration during this period will impact on brain development.

Neonatal alcohol exposure has been shown to cause cerebellar defects (Eluwa, 2008). If neonatal alcohol exposure can affect the development of cerebellum, it is likely that other parts of the brain like the hypothalamus can be affected leading to disruption in hypothalamic-pituitary-gonadal (HPG) axis regulation. It is also possible that postnatal alcohol exposure could affect brain development if exposure coincides with the period of maximal vulnerability to environmental insults. This could also lead to marked effects on the development of HPG axis which plays a critical role in the control of reproduction (Young and Heath, 2004). This effect on HPG disrupts hormone release from pituitary gland and hypothalamus which can cause problems for male reproductive and sexual functions (Hewit, 2014). Male rat offspring whose dams ingested alcohol during late pregnancy display atypical sexual behaviour in adulthood (Ingeborg et al., 2003). This study attempts to investigate the effects of gestational and/or lactational alcohol exposure on the histomorphmetry of the testes in neonatal rats.

\section{MATERIALS AND METHODS Experimental animals}

Seventy five female Wistar rats aged 4 - 5 weeks were used in this investigation. The rats were obtained from the Laboratory Animal Unit, Faculty of Veterinary Medicine, University of Ibadan, Nigeria. All the animals were fed water and commercial diet (Guinea Feed) ad libitum throughout the duration of the study. After acclimatization for five weeks, the 75 female rats were divided into 3 groups of 25 each. The offsprings of group 1 served as control (C), those of group 2 were exposed to alcohol during pregnancy and lactation (APL) while those of group 3 were exposed to alcohol during lactation only (AL).

\section{Breeding of experimental animals}

At the commencement of the study, the female rats weighing 145-170 g were bred overnight by introducing 1 male rat into a cage housing 5 female rats. Day 1 of 
pregnancy was presumed after observing vaginal/copulation plug the following morning (Onu and Ezeasor, 2001)

\section{Alcohol administration}

Following pregnancy detection, $2 \mathrm{~g} / \mathrm{kg}$ body weight of $30 \%$ ethanol (v/v) was given to the pregnant rats in APL per os (Maier and West, 2004). This continued throughout pregnancy and lactation. After delivery, the lactating rats in $\mathrm{AL}$ were given the same quantity of ethanol per os and this lasted throughout lactation period. The control rats were given equal volume of distilled water.

\section{Sample collection and histopathological evaluation}

At D7, 14, 21, 35 and 49 postpartum, 5 male offsprings from the 3 groups were weighed and sacrificed by cervical dislocation. After the sacrifice, testes were dissected out and weighed using Metler's Analytical Balance (MICROWA SWISS 5540).

Thereafter, the testicular tissues were dehydrated through a graded series of ethanol (50\%, 70\%, 90\%, and 100\%) (Junqueira and Carneiro, 2003). The dehydration using graded series of alcohol was to gradually extract water from the tissue. Clearing means the replacement of the ethanol with xylene which is miscible with the infiltrating and embedding medium which is paraffin in molten form. The molten paraffin when solidifies, hardens the tissue to enable sections to be cut. Sections (5-6 $\mu \mathrm{m}$ in thickness) were stained with haematoxylin and eosin (H\&E). Haematoxylin is a basic dye and therefore stains acidic structures of tissue purplish blue. Acidic structures include nuclei, ribosomes and rough endoplamic reticulum which have high content of deoxyribonucleic acid (DNA) and ribonucleic acid (RNA). In contrast, eosin is an acidic dye and stains basic structures red or pink. Most cytoplamic proteins are basic and hence cytoplasm usually stains pink or red (Young and Heat, 2004). The sections were then evaluated for histopathological changes under the light microscope. The diameter of 5 randomly selected circular or nearly circular profiles of seminiferous tubules of the testes were measured using calibrated eyepiece micrometer $(\mu \mathrm{m})$. Photomicrographs were thereafter produced.

\section{Statistical analysis}

The data generated from all the measurements were subjected to statistical analyses. Means and standard errors of means (means \pm SEM) were calculated for each group. Statistical differences between the mean values of the diameter of seminiferous tubules of the testes were analyzed using One-way analysis of variance (ANOVA) and Duncan's Multiple Range Test (DMRT) (Duncan, 1955). For the statistical analysis, $\mathrm{P}<0.05$ was considered statistically significant.

\section{RESULTS}

At D49, the testes of APL and AL contained profiles of seminiferous tubules with few adluminal germ cells as well as clumps of defoliated cytogenic cells, while the seminiferous tubules of control testes showed normal active epithelium Figure 1.

As shown on Table 1, the seminiferous tubular diameter of the testes in APL were significantly reduced $(\mathrm{p}<0.05)$ relative to the control throughout the duration of the study while those of AL were significantly reduced $(p<0.05)$ at D7, 21, 35 and 49 relative to the control. At D14, the seminiferous tubular diameter of the testes in $\mathrm{AL}$ were similar ( $p>0.05)$ to the control. 

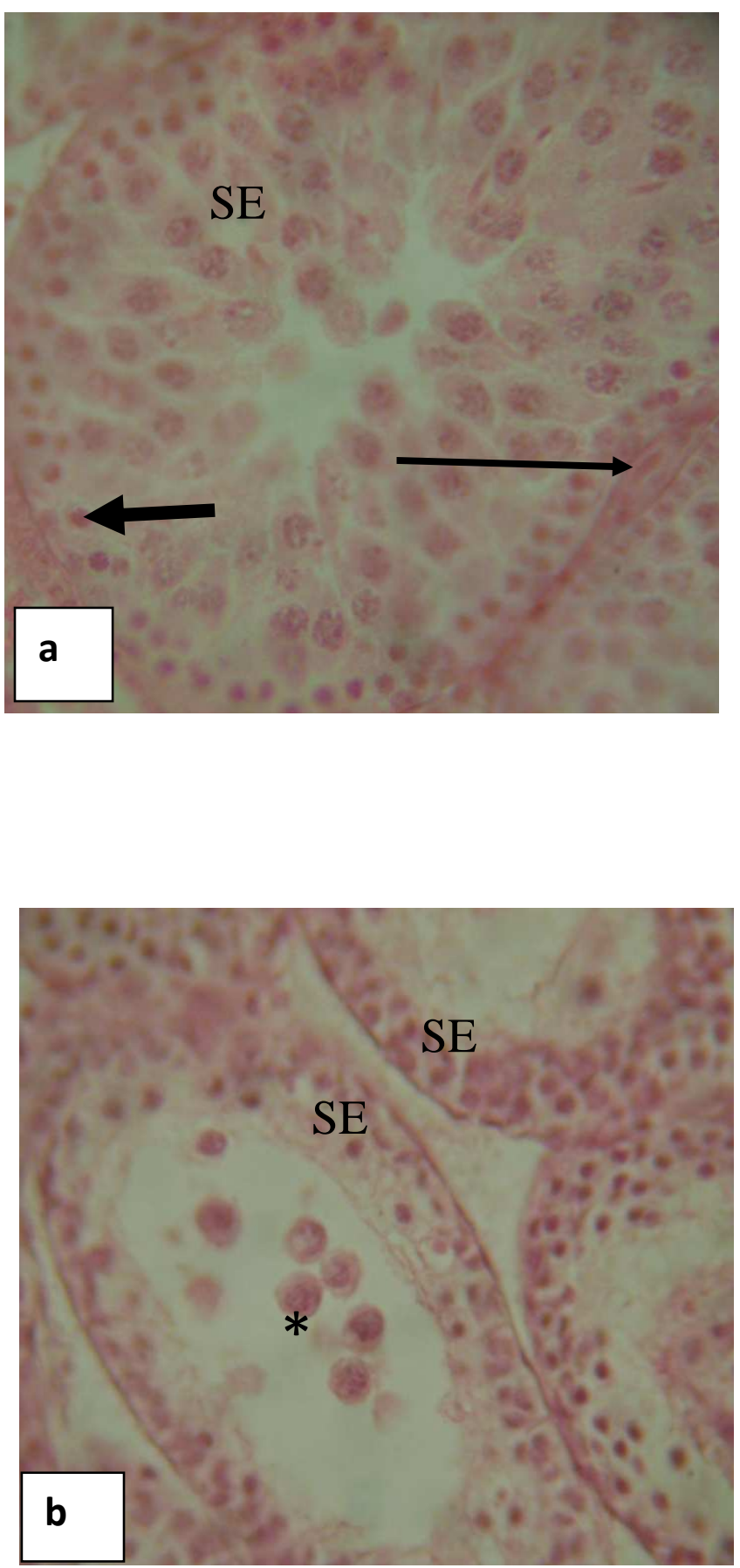

Figure 1: Photomicrograph sections of testes of Wistar rat offspring (D49).

(a) Control showing active epithelium. (b) APL (D49) showing reduced number and clumps of defoliated spermatogenic epithelium (*). Similar observation was made in AL (D49). SE = Seminiferous epithelium, Sertoli cell (thick arrow), Myoid cell (thin arrow ). Magnification:x600 Stain= H\&E. 
Table 1: Mean ( \pm SEM) diameter $(\mu \mathrm{m})$ of seminiferous tubules of testes of Wistar rat offsprings whose dams were exposed to alcohol during gestation and/or lactation.

\begin{tabular}{lccc}
\hline $\begin{array}{l}\text { Period of lactation } \\
(\mathbf{D})\end{array}$ & Control $(\mathbf{n}=\mathbf{5})$ & APL $(\mathbf{n}=\mathbf{5})$ & AL $(\mathbf{n}=\mathbf{5})$ \\
\hline 7 & $50.00 \pm 0.32^{\mathrm{a}}$ & $38.00 \pm 0.32^{\mathrm{c}}$ & $41.00 \pm 0.32^{\mathrm{b}}$ \\
14 & $61.00 \pm 0.55^{\mathrm{a}}$ & $40.00 \pm 0.32^{\mathrm{c}}$ & $52.00 \pm 0.32^{\mathrm{a}}$ \\
21 & $82.00 \pm 0.63^{\mathrm{a}}$ & $53.00 \pm 0.45^{\mathrm{c}}$ & $68.00 \pm 0.32^{\mathrm{b}}$ \\
35 & $129.00 \pm 0.32^{\mathrm{a}}$ & $45.00 \pm 0.32^{\mathrm{c}}$ & $110.00 \pm 0.63^{\mathrm{b}}$ \\
49 & $180.00 \pm 0.32^{\mathrm{a}}$ & $94.00 \pm 0.32^{\mathrm{c}}$ & $140.00 \pm 0.32^{\mathrm{b}}$ \\
\hline \multicolumn{4}{l}{ Values in the same row with different superscripts are significantly different at p<0.05. } \\
APL = Male rat offspring whose dams were exposed to alcohol from pregnancy to lactation; \\
AL= Male rat offspring whose dams were exposed to alcohol during lactation only; \\
n = number of animals.
\end{tabular}

\section{DISCUSSION}

This investigation showed that alcohol administration during pregnancy and/or lactation caused disruption of spermatogenic process and reduced diameter of seminiferous tubules of testes in Wistar rat offsprings. This disruption of spermatogenesis and reduced seminiferous tubular diameter observed on the testes of offspring whose dams were exposed to alcohol from pregnancy to lactation in this investigation is similar to the observation of Onu et al. (2003) in testes of mice. However, information on the effects of alcohol on the testes of offspring whose dams were exposed to alcohol during lactation only is scarce in the literature and it is therefore being documented. The mechanism of this effect of alcohol on the testes of Wistar rat offspring whose dams were exposed to alcohol from pregnancy to lactation and also during lactation only was not immediately known. However, there are some possible explanations that could account for this.

In vitro study using embryonic and 3week old testes by Li and Kim (2003) showed that alcohol enhances germ cell (gonocytes) apoptosis. At D7 of postnatal life (micrograph not shown) in this investigation, there were degenerations of germ cells (gonocytes) which could be due to apoptosis being enhanced by alcohol administered to the dams from pregnancy to lactation and during lactation only. In animals, there is evidence that alcohol exposure of adults can increase germ cell apoptosis (Zhu et al., 2000) and cause an adverse effect on the secretory function of Sertoli cells (Eid et al., 2013). This in vivo investigation demonstrated that alcohol causes germ cell (gonocytes) death. Apoptosis during this neonatal period may result in abnormal spermatogenesis in adults because it has been reported that normal development of germ cells in the first 3-5 postnatal days is critical to healthy spermatogenesis later in life (Adams and McLaren, 2002). It therefore implies that the few germ cells in the testes whose dams were exposed to alcohol from pregnancy to lactation and during lactation only could be due to toxic effect of alcohol on the germ cells early in life.

The other explanation is toxicity of alcohol on tissues. Passage of alcohol from the dam to the foetus occurs via the placenta to a point where foetal and maternal concentrations are almost equal (Gauthier et al., 2004). Alcohol has direct toxic effect on foetal organs development (Costa et al., 2004). Alcohol administered to lactating dams is transferred to neonates through the mammary gland milk (Mennella, 2001). Neonates have limited capacity to metabolize alcohol which in turn may accumulate in the tissues. This accumulation may be due to lower activity of enzyme in the liver called 
cytochrome P-450 system and alcohol dehydrogenase which are involved in alcohol metabolism (Mennella, 2001). This accumulated alcohol could be destructive to the tissues of the foetus leading to poor growth of the body and organs including the testes.

Additionally, alcohol is a known neurotoxin (Costa et al., 2004) and could destroy the developing brain including possibly those of the hypothalamus. Alcohol exposure during the brain growth spurt which is characterized by proliferation of glial cells and maturation of neurons leads to microcephaly (Costa et al., 2004). In this investigation, alcohol was administered to dams from pregnancy to lactation and during lactation only. It is possible therefore that the alcohol might have affected the development of the brain including the hypothalamus. The hypothalamus has neurosecretory neurons which synthesize and release hypothalamic releasing factors that act on rostral pituitary gland to release luteinizing hormone (LH), follicule-stimulating hormone (FSH) and interstitial cell stimulating hormone (ICSH) (Young and Heath, 2004). It has been observed that prenatal alcohol alters endocrine functions such as hypothalamic-pituitarygonadal axis in the offspring (Schwartz and Carey, 2005). It is also possible that postnatal alcohol could disrupt the HPG axis regulation of the offspring because the days of administration coincided with the period of maximal vulnerability of the brain to environmental insults such as alcohol. Gonadotropic hormones control the maturation and regulation of the action of the gonads including growth and hormone production (Hewit, 2014). It is therefore reasonable to suggest that the inhibition of spermatogenesis and reduction in seminiferous tubular diameter could be as result of toxic effect of alcohol on the testes as well as defective gonadotropic secretion and release resulting from the disruption of HPG axis regulation as earlier suggested by Onu and Ezeasor (2001).

\section{Conclusion}

The results of this investigation indicated that alcohol consumption during pregnancy and/or lactation had adverse effect on testicular growth and spermatogenesis. Thus the public should be enlightened regarding the effects of alcoholic beverages used during pregnancy and/or lactation, and the possible adverse outcome associated with prenatal alcohol exposure. This will go a long way to ensuring that children are guarded against these adverse outcomes.

\section{REFERENCES}

Adams RI, McLaren A. 2002. Sexually dimorphic development of mouse primordial germ cells: switching from oogenesis to spermatogenesis. Development., 129: 1156-1164.

Astley SJ. 2004. Diagnostic Guide for Fetal Alcohol Spectrum Disorders: The 4Digit Diagnostic Code. Seattle: University of Washington. Retrieved on $4 / 11 / 2007$.

Beaulieu C, Rasmassen C. 2014. Fetal alcohol spectrum disorder impacts brain development throughout childhood and adolescence not just at birth. Retrieved from www.sciencedaily.com on $19 / 2 / 14$.

Berma R. 2014. Prenatal alcohol exposure found to affect key developmental brain proteins. Retrieved on 21/2/14 from www.ucdmc.ucdavis.edu

Costa LG, Vitalone A, Guizzetti M. 2004. Signal transduction mechanisms involved in the antiproliferative effects of ethanol in glial cells. Tox. Let., 149(2-3): 67-73.

Duncan DB. 1955. Multiple Range and Multiple Fishers Tests. Biometrics., II: $1-42$.

Eid N, Ito U, Otsuki Y. 2013. Anti-Apoptotic Mechanisms of Sertoli cells against 
Ethanol Toxicity: Alcohol and Drug Dependence. Alcohol Dependence., 1(1): 1-2.

Eluwa M, Njoku C, Ekanem T, Akpantah A. 2008. Teraogenic effect of beer and palm wine on histology of fetal cerebral cortex of Wistar Rats. The Internet J. Hlth., 9(1).

Gauthier WT, Ping X-D, Harris FL,Wong M, Elbabesh H Brown LAS. (2004. Fetal alcohol exposure impairs alveolar macrophage function via decreased glutathione availability. Ped. Res., 57: 76-81.

Hewit R. 2014. How does alcohol affect the reproductive system? Retrieved from www.livestrong.com on 20/2/14.

Ingeborg LW, Ward BO, Affuso JD, Long 111 WD, French JA, Hendricks SE. 2003. Fetal testosterone surge: specific modulations induced in male rats by maternal stress/or alcohol consumption. Horm. Beh., 43: 531-539.

Junqueira LC, Carneiro J. 2003. Basic Histology (10 ${ }^{\text {th }}$ edn). Text and Atlas. Lange International: New York.

Lam MKP, Homewood J, Taylor AJ, Mazurski EJ. 2000. Second generation effects of maternal alcohol consumption during pregnancy in rats. Prog.Neuro-Psychopharm. Biol.Psych., 24: 619-631.

Li H, Kim KH. 2003. Effect of ethanol on embryonic and neonatal rat testes in organ culture. J. Androl., 24(5):1-15.

Maier SE, West JR. 2004. Patterns of alcoholrelated birth defects. National Institute on Alcohol Abuse and Alcoholism. Bethesda, Maryland. Retrieved January 1, 2005 from http: //www. niaaa.nih. gov
May PA, Gossage JP. 2001. "Estimating the prevalence of fetal alcohol syndrome. A summary." Alcohol Res. Hlth., 25(3): 159-67.

Mennella J. 2001. Alcohol's effects on lactation. NIAAA, Bethesda, Maryland. Retrieved January 14, 2005 from http://www.niaaa.nih.gov

Onu JE. Ezeasor DN. 2001. Retardation of testicular growth in mice in FAS: Suspected evidence $f$ disruption of hypothalamic-pituitary-gonadal axis regulation. Nig. Vet. J., 22(2): 35-41.

Onu JE, Ezeasor DN, Ihemelandu EC. 2003. Histological evidence of retardation of spermatogenesis in mice in experimentally induced Foetal Alcohol Syndrome. Pakistan J. Biol. Sci., 6(22): 1860-1863.

Schwartz J, Carey LC. 2005. Programming effect of moderate and binge alcohol consumption. In Early life Origins of Health and Disease, Wintour-Coughlan EM, Ovens J (eds); 1-8.

Spear L. 2000. Modeling adolescent development and alcohol use in animals. Alcohol Res. Hlth., 24(2): 115-123.

Young B, Heath JW. 2004. Wheater's Functional Histology: a Text and Colour Atlas (4 ${ }^{\text {th }}$ edn). Churchill Livingstone: Edinburgh.

Zhu Q, Van Thiel DH, Gavaler JS. 2006. Effects of Ethanol rat Sertoli cell function. Studies in vitro and in vivo. Alcohol Clin. Exp. Res., 24: 1550-1556. 